\title{
Antithrombin III reduces collagen-stimulated granule secretion of PDGF-AB and the release of soluble CD40 ligand from human platelets
}

\author{
TOMOAKI DOI $^{1,2}$, SEIJI ADACHI ${ }^{2}$, RIE MATSUSHIMA-NISHIWAKI ${ }^{2}$, HISAAKI KATO ${ }^{1,2}$, YUKIKO ENOMOTO $^{3}$, \\ HIDEO NATSUME ${ }^{2,4}$, KENJI KATO ${ }^{2,4}$, JUN MIZUTANI ${ }^{4}$, TAKANOBU OTSUKA ${ }^{4}$, HARUHIKO TOKUDA ${ }^{5}$, \\ SHIGERU AKAMATSU ${ }^{6}$, TORU IWAMA ${ }^{3}$, OSAMU KOZAWA ${ }^{2}$ and SHINJI OGURA ${ }^{1}$
}

\begin{abstract}
Departments of ${ }^{1}$ Emergency and Disaster Medicine, ${ }^{2}$ Pharmacology, and ${ }^{3}$ Neurosurgery, Gifu University
Graduate School of Medicine, Gifu 501-1194; ${ }^{4}$ Department of Orthopedic Surgery, Nagoya City University

Graduate School of Medical Sciences, Nagoya $467-8601 ;{ }^{5}$ Department of Clinical Laboratory,

National Hospital for Geriatric Medicine, National Center for Geriatrics and Gerontology, Obu 474-8511;

${ }^{6}$ Department of Intensive Care Medicine, Matsunami General Hospital, Gifu 501-6062, Japan
\end{abstract}

Received April 8, 2010; Accepted June 3, 2010

DOI: 10.3892/ijmm_00000477

\begin{abstract}
Although antithrombin-III (AT-III), an anticoagulant, has been shown to affect human platelet functions, the direct effect of AT-III on platelets is still unknown. We recently reported that the collagen-induced phosphorylation of the heat shock protein 27 (HSP27) via the p44/p42 mitogen-activated protein (MAP) kinase is sufficient for granule secretion and the release of soluble CD40 ligand (sCD40L) from platelets but not platelet aggregation. In the present study, we investigated whether AT-III affects the collagen-induced secretion of the platelet-derived growth factor (PDGF)-AB and SCD40L release. AT-III inhibited collagen-stimulated platelet aggregation. The collageninduced secretion of PDGF-AB was significantly suppressed by AT-III. AT-III also reduced SCD40L release. AT-III markedly attenuated the collagen-induced phosphorylated levels of p44/p42 MAP kinase. In addition, AT-III suppressed collagen-induced HSP27 phosphorylation. These results strongly suggest that AT-III reduced collagen-stimulated platelet granule secretion due to the inhibition of HSP27 phosphorylation via p44/p42 MAP kinase.
\end{abstract}

\section{Introduction}

It is generally recognized that antithrombin III (AT-III), a member of the serpin family, is one of the most important

Correspondence to: Dr Osamu Kozawa, Department of Pharmacology, Gifu University Graduate School of Medicine, Gifu 501-1194, Japan

E-mail: okozawa@gifu-u.ac.jp

Key words: antithrombin-III, collagen, platelet-derived growth factor- $\mathrm{AB}$, soluble $\mathrm{CD} 40$ ligand, platelet physiological inhibitors of coagulation such as thrombin $(1,2)$. AT-III itself slowly prevents thrombin-activity, but when heparin and heparan sulfate proteoglycans exist on microvascular and macrovascular endothelial cells, the inactivating ability for thrombin is further enhanced. AT-III is clinically used as a therapeutic for sepsis or septic shock with disseminated intravascular coagulation and plays a pivotal role in coagulation abnormality (3). In contrast to heparin, which prevents coagulopathy, but does not reduce overall mortality, AT-III significantly increases the survival of endotoxin-induced shock without improving coagulopathy $(4,5)$. Accumulating evidence suggests that AT-III demonstrates not only an anticoagulant effect but also an antiinflammatory effect. It has recently been shown that AT-III directly affects platelets, resulting in the elicitation of the anti-inflammatory effect (6). However, the exact mechanism of AT-III in platelets remains to be clarified.

It is well recognized that collagen is a pivotal activator for human platelets, and its receptors on platelet membranes are mainly classified into glycoprotein VI (GPVI) and integrin $\alpha 2 \beta 1$ (7). The firm adhesion of activated integrin $\alpha 2 \beta 1$ by binding to collagen and strengthening of GPVIcollagen interactions leads to integrated signaling and the further up-regulation of integrin activity, resulting in the enhancement of granule secretion, and the development of coagulant activity (8-10). Heat shock proteins (HSPs) are expressed in a variety of cells when exposed to environmental stress such as heat and chemicals (11). HSPs are classified into high-molecular-weight HSPs such as HSP90 and HSP70, and low-molecular-weight HSPs such as HSP27 and $\alpha \mathrm{B}$-crystallin, based on their apparent molecular sizes. Low-molecular-weight HSPs with molecular masses from 10 to $30 \mathrm{kDa}$ have high homology in their amino acid sequences (12). Low-molecular-weight HSPs may have chaperoning functions similar to the high-molecular-weight HSPs (12).

It is recognized that HSP27 activity is regulated by posttranslational modifications such as phosphorylation $(12,13)$. 
HSP27 becomes rapidly phosphorylated in response to various stresses, as well as exposure to cytokines and mitogens $(14,15)$. Human HSP27 is phosphorylated at three serine residues (Ser-15, Ser-78 and Ser-82). While HSP27 exists in an aggregated form under unstimulated conditions, it is rapidly dissociated as a result of stimulation-responsive phosphorylation. It has been shown that HSP27 phosphorylation is catalyzed by the MAP kinase superfamily such as p38 MAP kinase, the stress-activated protein kinase/c-Jun $N$-terminal kinase (SAPK/JNK) and p44/p42 MAP kinase $(16,17)$. It has been reported that collagen regulates the phosphorylation of HSP27, which is most likely required for human platelet activation (18). We recently showed that in human platelets the collagen-induced phosphorylation of HSP27 via p44/p42 MAP kinase is correlated with platelet granule secretion such as the platelet-derived growth factor (PDGF)-AB and the release of soluble CD40 ligand (sCD40L), but not for platelet aggregation (19). However, the exact mechanism of collagen in platelet activation is not precisely clarified.

In the present study, we examined the effect of AT-III on collagen-induced platelet granule secretion and its exact mechanism. We show that AT-III suppresses the collageninduced secretion of PDGF-AB from the human platelet granule and the release of $\mathrm{sCD} 40 \mathrm{~L}$ due to the inhibition of HSP27 phosphorylation via p44/p42 MAP kinase.

\section{Materials and methods}

Materials. Collagen was purchased from Nycomed Pharma GmbH (Munich, Germany). AT-III was a generous gift from CSL Behring (Tokyo, Japan). AT-III was extracted from human blood and its purity was $>97.9 \%$ (analyzed by High Performance Liquid Chromatography) or $>98.2 \%$ [analyzed by sodium dodecyl sulfate (SDS)-polyacrylamide gel electrophoresis (PAGE)]. AT-III was stored as a freeze-dried agent and dissolved with distilled water. PDGF-AB enzymelinked immunosorbent assay (ELISA) kits and sCD40L ELISA kits were purchased from R\&D System, Inc. (Minneapolis, MN). Phospho-specific p44/p42 MAP kinase and p44/p42 MAP kinase antibodies were obtained from Cell Signaling, Inc. (Beverly, MA). Phospho-specific HSP27 (Ser-15) and phospho-specific HSP27 antibodies (Ser-78) were from Stressgen Biotechnologies (Victoria, BC, Canada). Phospho-specific HSP27 antibodies (Ser-82) were from Biomol Research Laboratories (Plymouth Meeting, PA). The other materials and chemicals were obtained from commercial sources.

Preparation of platelets. Human blood was donated from healthy volunteers into a $1 / 10$ volume of $3.8 \%$ sodium citrate. Platelet-rich plasma (PRP) was obtained from blood samples by centrifugation at $155 \mathrm{x} \mathrm{g}$ for $12 \mathrm{~min}$ at room temperature. Platelet-poor plasma was prepared from residual blood by centrifugation at 2,500 $\mathrm{x}$ g for $5 \mathrm{~min}$. All participants signed an informed consent after receiving a detailed explanation and the study was approved by the Committee of Ethics of the Gifu University Graduate School of Medicine.

Measurement of platelet aggregation induced by collagen. Platelet aggregation using citrated PRP was carried out in a
PA-200 aggregometer (Kowa Co. Ltd., Tokyo, Japan), which can determine the size of platelet aggregates based upon particle counting using laser scattering methods (small size, 9-25 $\mu \mathrm{m}$; medium size, 25-50 $\mu \mathrm{m}$; large size, 50-70 $\mu \mathrm{m}$ ) (20), at $37^{\circ} \mathrm{C}$ with a stirring speed of $800 \mathrm{rpm}$. The platelets were preincubated for $1 \mathrm{~min}$, and then platelet aggregation was monitored for $4 \mathrm{~min}$. The percentage of transmittance of the isolated platelets was recorded as $0 \%$, and that of the appropriate platelet-poor plasma (blank) was recorded as $100 \%$. When indicated, PRP was pretreated with AT-III for $15 \mathrm{~min}$.

Protein preparation after collagen stimulation. After the stimulation with collagen, platelet aggregation was terminated by the addition of ice-cold EDTA $(10 \mathrm{mM})$ solution. The mixture was centrifuged at $10,000 \mathrm{xg}$ at $4^{\circ} \mathrm{C}$ for $2 \mathrm{~min}$. To measure PDGF-AB and $\mathrm{sCD} 40 \mathrm{~L}$ as described below, the supernatant was isolated and stored at $-20^{\circ} \mathrm{C}$ for subsequent ELISA. For Western blot analysis, the pellet was washed twice with phosphate-buffered saline and then lysed and immediately boiled in a lysis buffer containing $62.5 \mathrm{mM}$ Tris/Cl, pH 6.8, 2\% SDS, $50 \mathrm{mM}$ dithiothreitol, and $10 \%$ glycerol.

Measurement of PDGF-AB and $s C D 40 L$ levels. The PDGF$\mathrm{AB}$ and $\mathrm{sCD} 40 \mathrm{~L}$ levels in samples were determined using each kit following the manufacturer's instructions.

Western blot analysis. Western blot analysis was performed as described previously (21). Briefly, SDS-PAGE was performed by the method of Laemmli (22) in a $10 \%$ or $12 \%$ polyacrylamide gel. The proteins in the gel were transferred onto a polyvinylidine fluoride (PVDF) membrane. The membranes were then blocked with $5 \%$ fat-free dry milk in Tris-buffered saline with $0.1 \%$ Tween-20 (TBS-T, $20 \mathrm{mM}$

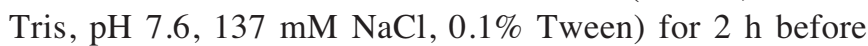
incubation with the indicated primary antibodies. The antibodies used in these studies were phospho-specific p44/p42 MAP kinase, p44/p42 MAP kinase, phospho-specific HSP27 (Ser-15), phospho-specific HSP27 (Ser-78), phospho-specific HSP27 (Ser-82), and HSP27 antibodies or GAPDH, respectively. Peroxidase-labeled anti-mouse $\operatorname{IgG}$ or antirabbit IgG antibodies were used as secondary antibodies. The first and second antibodies were diluted for optimum concentration respectively, with 5\% fat-free dry milk in TBS-T. Peroxidase activity on PVDF membranes was visualized on X-ray film by means of an ECL Western blotting detection system following the manufacturer's protocol.

Statistical analysis. The data were analyzed by the Student's t-test, and a $\mathrm{p}<0.05$ was considered to be significant. All data are presented as the mean \pm SEM.

\section{Results}

Effect of AT-III on platelet aggregation by collagen stimulation. We first examined the effect of AT-III on platelet aggregation stimulated by collagen using a platelet aggregometer. AT-III markedly reduced the collagenstimulated platelet aggregation in a dose-dependent manner 


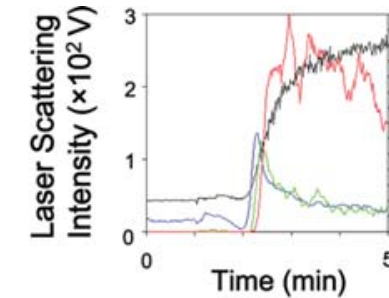

panel

1
0

AT-III (U/ml)

collagen

Ratio (\%)

\begin{tabular}{|c|c|c|c|c|}
\hline small & 20 & 18 & 27 & 57 \\
\hline medium & 15 & 18 & 19 & 28 \\
\hline large & 65 & 63 & 54 & 16 \\
\hline total & 100 & 100 & 100 & 100 \\
\hline
\end{tabular}

Figure 1. Effect of AT-III on the collagen-induced platelet aggregation. PRP was pretreated with various doses of AT-III at $37^{\circ} \mathrm{C}$ for 15 min, and stimulated by $1.0 \mathrm{mg} / \mathrm{ml}$ collagen for $5 \mathrm{~min}$. The reaction was terminated by the addition of ice-cold EDTA (10 mM) solution. Black line indicates the percentage of transmittance of each sample (the isolated platelets were recorded as $0 \%$, and platelet-free plasma was recorded as $100 \%$ ). Blue line indicates small aggregates $(9-25 \mu \mathrm{m})$; green line, medium aggregates $(25-50 \mu \mathrm{m})$; red line, large aggregates $(50-70 \mu \mathrm{m})$. The distributions $(\%)$ of aggregated particle sizes were measured using laser scattering methods. Representative results obtained from five healthy donors are indicated.

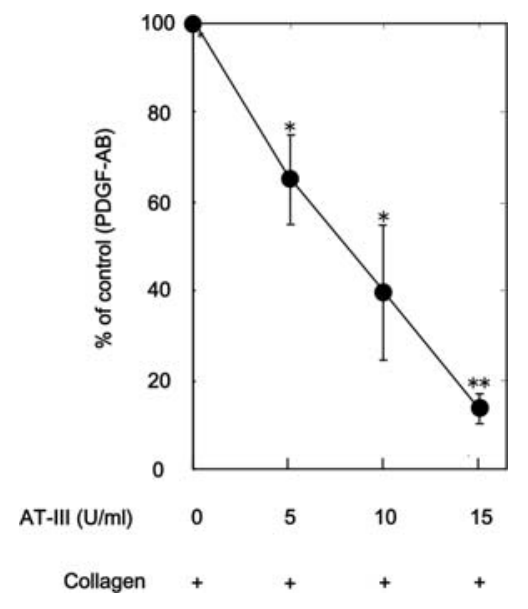

Figure 2. Effect of AT-III on the collagen-induced PDGF-AB secretion in human platelets. PRP was pretreated with various doses of AT-III at $37^{\circ} \mathrm{C}$ for $15 \mathrm{~min}$, and stimulated by $1.0 \mathrm{mg} / \mathrm{ml}$ collagen for $30 \mathrm{~min}$. The reaction was terminated by the addition of ice-cold EDTA $(10 \mathrm{mM})$ solution. The mixture was centrifuged at $10,000 \mathrm{xg}$ at $4^{\circ} \mathrm{C}$ and the supernatants were then subjected to ELISA for PDGF-AB. The net increased levels of collagen alone are represented as $100 \%$. Representative results from five independent experiments are shown. Each value represents the mean \pm SEM. $* \mathrm{p}<0.05$, compared with the value of the control.

in the range between 5 and $15 \mathrm{U} / \mathrm{ml}$ (Fig. 1) as previously shown (6). AT-III dose-dependently decreased large aggregates $(50-70 \mu \mathrm{m})$ according to the analysis of the size of platelet aggregates (Fig. 1). On the contrary, small aggregates $(9-25 \mu \mathrm{m})$ and medium aggregates $(25-50 \mu \mathrm{m})$ were markedly increased by AT-III (Fig. 1).

Effect of AT-III on the collagen-induced platelet granule secretion. In order to investigate whether AT-III affects collagen-induced human platelet granule secretion, we next examined the effect of AT-III on the collagen-induced secretion of PDGF-AB from platelets. We found that collagen stimulated the secretion of PDGF-AB $(255 \pm 20 \mathrm{pg} / \mathrm{ml}$ for control; $8990 \pm 1050 \mathrm{pg} / \mathrm{ml}$ for $1.0 \mathrm{mg} / \mathrm{ml}$ collagen, as measured during the stimulation for $30 \mathrm{~min})$. AT-III (15 U/ml) significantly suppressed the collagen-induced PDGF-AB secretion from human platelets in a dose-dependent manner (Fig. 2). AT-III markedly inhibited the PDGF-AB secretion and $15 \mathrm{U} / \mathrm{ml}$ of AT-III caused an $\sim 80 \%$ reduction in the collagen effect (Fig. 2).

Effect of AT-III on the collagen-stimulated release of SCD4OL from human platelets. We next examined the effect of AT-III on the collagen-stimulated sCD40L release from platelets. We found that collagen stimulated the release of $\mathrm{sCD} 40 \mathrm{~L}$ $(63 \pm 10 \mathrm{pg} / \mathrm{ml}$ for control; $4573 \pm 250 \mathrm{pg} / \mathrm{ml}$ for $1.0 \mathrm{mg} / \mathrm{ml}$ collagen, as measured during the stimulation for $30 \mathrm{~min}$ ). AT-III significantly inhibited the collagen-stimulated release of sCD40L (Fig. 3). The suppressive effect of AT-III was dose-dependent and $15 \mathrm{U} / \mathrm{ml}$ of AT-III caused an $~ 80 \%$ reduction in the collagen effect (Fig. 3). 


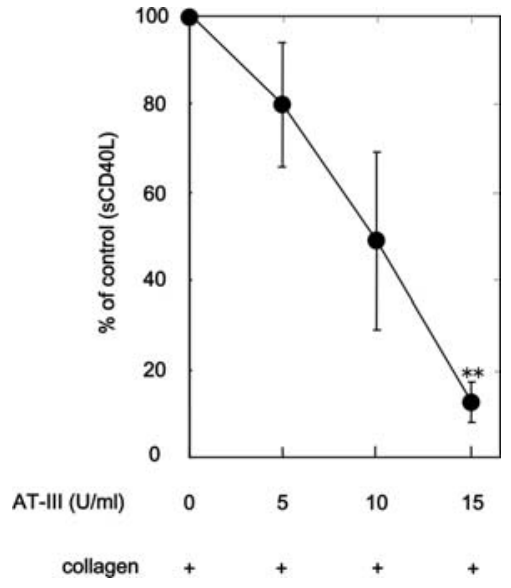

Figure 3. Effect of AT-III on the collagen-induced sCD40L release in human platelets. PRP was pretreated with various doses of AT-III at $37^{\circ} \mathrm{C}$ for $15 \mathrm{~min}$, and stimulated by $1.0 \mathrm{mg} / \mathrm{ml}$ collagen for $30 \mathrm{~min}$. The reaction was terminated by the addition of ice-cold EDTA $(10 \mathrm{mM})$ solution. The mixture was centrifuged at $10,000 \mathrm{xg}$ at $4^{\circ} \mathrm{C}$, and the supernatants were then subjected to ELISA for sCD40L. The net increased levels of collagen alone are represented as $100 \%$. Representative results from five independent experiments are shown. Each value represents the mean \pm SEM. ${ }^{*} p<0.05$, compared with the value of the control.

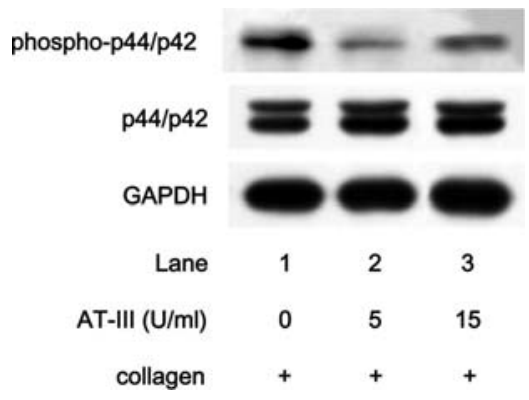

Figure 4. Effect of AT-III on the collagen-induced phosphorylation of p44/p42 MAP kinase in human platelets. PRP was pretreated with the indicated doses of AT-III at $37^{\circ} \mathrm{C}$ for $15 \mathrm{~min}$, and stimulated by $1.0 \mathrm{mg} / \mathrm{ml}$ collagen for $5 \mathrm{~min}$. The reaction was terminated by the addition of ice-cold EDTA $(10 \mathrm{mM})$ solution. Lysed platelets were subjected to Western blot analysis using antibodies against phospho-specific p44/p42 MAP kinase, p44/p42 MAP kinase or GAPDH. Representative results from at least three independent experiments are shown.

Effect of AT-III on the collagen-induced phosphorylation of p44/p42 MAP kinase or HSP27 in human platelets. We previously showed that collagen stimulates the phosphorylation of HSP27 via p44/p42 MAP kinase in human platelets, and subsequently induces the secretion of PDGF-AB and the release of sCD40L (19). Therefore, we next examined the effect of AT-III on the collagen-induced phosphorylation of p44/p42 MAP kinase in human platelets. AT-III markedly reduced the collagen-induced phosphorylation levels of $\mathrm{p} 44 /$ p42 MAP kinase (Fig. 4).

In our previous study (19), we demonstrated that collagen elicits HSP27 phosphorylation at three serine residues, Ser-15, Ser-78 and Ser-82, in human platelets. Furthermore, the effect of AT-III on the collagen-induced phosphorylation of HSP27 (Ser-15, Ser-78 and Ser-82) was examined. The

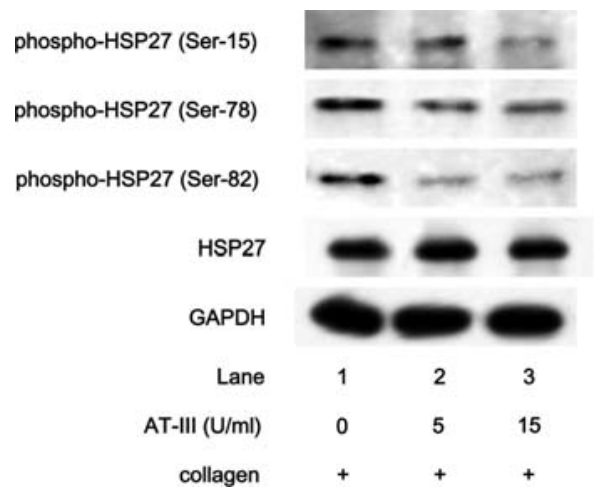

Figue 5. Effect of AT-III on the collagen-induced phosphorylation of HSP27 in human platelets. PRP were pretreated with the indicated doses of AT-III at $37^{\circ} \mathrm{C}$ for $15 \mathrm{~min}$, and then stimulated by $1.0 \mathrm{mg} / \mathrm{ml}$ collagen for $5 \mathrm{~min}$. The reaction was terminated by the addition of ice-cold EDTA $(10 \mathrm{mM})$ solution. Lysed platelets were subjected to Western blot analysis using antibodies against total HSP27, phospho-specific HSP27 (Ser-15, Ser-78 and Ser-82) or GAPDH. Representative results from at least three independent experiments are shown.

phosphorylation levels of HSP27 (Ser-15, Ser-78 and Ser-82) induced by collagen were markedly attenuated by AT-III (Fig. 5).

\section{Discussion}

In the present study, we focused on the effects of AT-III on collagen-induced human platelet functions. First, we found that AT-III significantly suppressed collagen-induced platelet aggregation and affected the distribution of aggregated particle sizes measured in laser scattering methods. We next investigated the effect of AT-III on collagen-induced granule secretion and showed that collagen-induced granule secretion of PDGF-AB was significantly inhibited by the pretreatment with AT-III. In addition, SCD40L release was also attenuated when the platelets were pretreated with AT-III. Since we recently reported that the collagen-induced phosphorylation of HSP27 via p44/p42 MAP kinase is positively correlated with platelet granule secretion such as PDGF-AB and the sCD40L release from platelets (19), we next examined the effects of AT-III on these phosphorylations. AT-III markedly suppressed the collagen-induced phosphorylation of p44/p42 MAP kinase and HSP27 at three serine residues, Ser-15, Ser-78 and Ser-82. Therefore, our results suggest that AT-III inhibits both granule secretion and SCD40L release by inhibiting HSP27 phosphorylation via p44/p42 MAP kinase in human platelets.

Platelets rapidly respond to a variety of stimuli and release the materials stored mainly in two specific granules, dense-granules and $\alpha$-granules, although platelets are anucleate cells that lack genomic DNA (23). These specific granule populations store different types of constituents. Between them, $\alpha$-granules contain large adhesive and healing proteins such as PDGF-AB and the von Willebrand factor (24). It is well recognized that PDGF-AB secreted from platelet $\alpha$-granules is a potent growth factor, which mainly acts on connective tissue (25). PDGF-AB induces the proliferation of vascular smooth muscle cells and plays a key 
role in the development of arteriosclerosis (25). In addition, activated platelets also release the inflammatory mediators of atherosclerosis, such as sCD40L. CD40L, a member of the tumor necrosis factor- $\alpha$ superfamily, is stored in the cytoplasm of resting platelets and is immediately translocated on the platelet membrane after activation by agonists such as ADP $(26,27)$. sCD40L is subsequently cleaved from the cell surface-expressed CD40L as a functional soluble fragment. It is generally known that the platelet-released sCD40L induces inflammatory responses via the CD40 expressed on vascular endothelial cells that produce inflammatory substances $(23,28)$. It has been shown that platelet-derived sCD40L becomes mobilized in acute coronary thrombotic indications (29). The elevation of plasma SCD40L is reportedly associated with an increased risk of cardiovascular events in patients with unstable coronary artery disease (30). In addition, it has been shown that sCD40L enhances the stimulation-induced platelet release of inflammatory substance from densegranules (31).

In the present study, we demonstrated that AT-III markedly suppressed the collagen-induced secretion of PDGF-AB, and the release of sCD40L from human platelets. Therefore, it is possible that inhibition by AT-III of sCD40L release from platelets plays a role in the immunosuppressive effect of this agent. Based on our findings as a whole, it is most likely that AT-III acts not only as an anticoagulant agent but also as an anti-inflammatory or anti-atherogenic agent through the reduction in the granule secretion of PDGF-AB and the release of sCD40L from platelets. AT-III is clinically used for the patient in critical state, such as severe sepsis or severe vascular disease (3). Taking our present findings into account, it is likely that AT-III could be effective to these patients not only as an anticoagulant agent but also as an anti-inflammatory agent. It has been recently reported that AT-III acts on platelet-membrane receptors directly, such as syndecan-4 (6). This report led us to speculate that AT-III acts on a certain platelet-membrane receptor, and subsequently suppresses the collagen signaling via inhibiting the phosphorylation of p44/p42 MAP kinase. Further investigation is required to clarify the exact mechanism underlying the AT-III-effects on human platelets.

In conclusion, our results strongly suggest that AT-III suppresses collagen-induced PDGF-AB secretion and the release of SCD40L due to the inhibition of HSP27 phosphorylation via $\mathrm{p} 44 / \mathrm{p} 42$ MAP kinase in platelets.

\section{Acknowledgements}

We are very grateful to Yoko Kawamura for her skillful technical assistance. This sudy was supported in part by a Grant-in-Aid for Scientific Research (nos. 20590565 and 20591825) from the Ministry of Education, Science, Sports and Culture of Japan and the Research Grants for Longevity Sciences (21A-3 and 21A-4) from the Ministry of Health, Labour and Welfare of Japan.

\section{References}

1. Bauer KA and Rosenberg RD: Role of antithrombin III as a regulator of in vivo coagulation. Semin Hematol 28: 10-18, 1991.
2. Levi M, de Jonge E and van der Poll T: Sepsis and disseminated intravascular coagulation. J Thromb Thrombolysis 16: 43-47, 2003.

3. Buller HR and ten Cate JW: Acquired antithrombin III deficiency: laboratory diagnosis, incidence, clinical implications, and treatment with antithrombin III concentrate. Am J Med 87: 44S-48S, 1989.

4. Triantaphyllopoulos DC: Effects of human antithrombin III on mortality and blood coagulation induced in rabbits by endotoxin. Thromb Haemost 51: 232-235, 1984.

5. Vinazzer H: Therapeutic use of antithrombin III in shock and disseminated intravascular coagulation. Semin Thromb Hemost 15: 347-352, 1989.

6. Kaneider NC, Feistritzer C, Gritti D, Mosheimer BA, Ricevuti G, Patsch JR and Wiedermann CJ: Expression and function of syndecan-4 in human platelets. Thromb Haemost 93: 1120-1127, 2005.

7. Kahn ML: Platelet-collagen responses: molecular basis and therapeutic promise. Semin Thromb Hemost 30: 419-425, 2004.

8. Shattil SJ and Newman PJ: Integrins: dynamic scaffolds for adhesion and signaling in platelets. Blood 104: 1606-1615, 2004.

9. Nieswandt B and Watson SP: Platelet-collagen interaction: is GPVI the central receptor? Blood 102: 449-461, 2003.

10. Moroi M and Jung S: Platelet grycoprotein VI: its structure and function. Thromb Res 114: 221-233, 2004.

11. Hendrick JP and Hartl FU: Molecular chaperone functions of heat-shock proteins. Annu Rev Biochem 62: 349-384, 1993.

12. Benjamin IJ and McMillan DR: Stress (heat shock) proteins: molecular chaperones in cardiovascular biology and disease. Circ Res 83: 117-132, 1998

13. Welch WJ: Phorbol ester, calcium ionophore, or serum added to quiescent rat embryo fibroblast cells all result in the elevated phosphorylation of two 28,000-dalton mammalian stress proteins. J Biol Chem 260: 3058-3062, 1985.

14. Gaestel M, Schroder W, Benndorf R, Lippmann C, Buchner K, Hucho F, Frdmann VA and Bielka H: Identification of the phosphorylation sites of the murine small heat shock protein hsp25. J Biol Chem 266: 14721-14724, 1991.

15. Landry J, Lambert H, Zhou M, Lavoie JN, Hickey E, Weber LA and Anderson CW: Human HSP27 is phosphorylated at serines 78 and 82 by heat shock and mitogen-activated kinases that recognize the same amino acid motif as S6 kinase II. J Biol Chem 267: 794-803, 1992.

16. Kyriakis JM and Avruch J: Sounding the alarm: protein kinase cascades activated by stress and inflammation. J Biol Chem 271 24313-24316, 1996.

17. Guay J, Lambert H, Gingras-Breton G, Lavoie JN, Huot J and Landry J: Regulation of actin filament dynamics by p38 map kinase-mediated phosphorylation of heat shock protein 27 . J Cell Sci 110: 357-368, 1997.

18. Saklatvala J, Rawlinson L, Waller RJ, Sarsfield S, Lee JC, Morton LF, Barnes MJ and Farndale RW: Role for p38 mitogen-activated protein kinase in platelet aggregation caused by collagen or a thromboxane analogue. J Biol Chem 271: 6586-6589, 1996.

19. Kato $H$, Adachi S, Doi $T$, Matsushima-Nishiwaki $R$, Minamitani C, Akamatsu S, Enomoto Y, Tokuda H, Otsuka T, Iwama $\mathrm{T}$, Kozawa $\mathrm{O}$ and Ogura $\mathrm{S}$ : Mechanism of collageninduced release of 5-HT, PDGF-AB and sCD40L from human platelets: role of HSP27 phosphorylation via p44/p42 MAPK. Thromb Res (In press).

20. Fabre JE, Nguyen M, Latour A, Keifer JA, Audoly LP, Coffman TM and Koller BH: Decreased platelet aggregation, increased bleeding time and resistance to thromboembolism in P2Y1-deficient mice. Nat Med 5: 1199-1202, 1999.

21. Kato K, Ito H, Hasegawa K, Inaguma $\mathrm{Y}$, Kozawa $\mathrm{O}$ and Asano T: Modulation of the stress-induced synthesis of hsp27 and alpha B-crystallin by cyclic AMP in C6 rat glioma cells. J Neurochem 66: 946-950, 1996.

22. Laemmli UK: Cleavage of structural proteins during the assembly of the head of bacteriophage. Nature 227: 680-685, 1970.

23. Davi G and Patrone C: Platelet activation and atherothrombosis. N Engl J Med 357: 2482-2492, 2007.

24. Rendu F and Brohard-Bohn B: The platelet release reaction: granules' constituents, secretion and functions. Platelets 12: 261-273, 2001. 
25. Heldin $\mathrm{CH}$ and Westermark B: Mechanism of action and in vivo role of platelet-derived growth factor. Physiol Rev 79: 1283-1316, 1999.

26. Hermann A, Rauch BH, Braun M, Schror K and Weber AA: Platelet CD40 ligand (CD40L) - subcellular localization, regulation of expression, and inhibition by clopidogrel. Platelets 12: 74-82, 2001 .

27. Andre P, Nannizzi-Alaimo L, Prasad SK and Phillips DR: Platelet-derived CD40L: the switch-hitting player of cardiovascular disease. Circulation 106: 896-899, 2002.

28. Henn V, Slupsky JR, Grafe M, Anagnostopoulos I, Forster R, Muller-Berghaus G and Kroczek RA: CD40 ligand on activated platelets triggers an inflammatory reaction of endothelial cells. Nature 391: 591-594, 1998.
29. Heeschen C, Dimmeler S, Hamm CW, van den Brand MJ, Boersma E, Zeiher AM and Simoons ML: Soluble CD40 ligand in acute coronary syndromes. N Engl J Med 348: 1104-1111, 2003.

30. Varo N, de Lemos JA, Libby P, Morrow DA, Murphy SA, Nuzzo R, Gibson CM, Cannon CP, Braunwald E and Schonbeck U: Soluble CD40L: risk prediction after acute coronary syndromes. Circulation 108: 1049-1052, 2003.

31. Zirlik A, Maier C, Gerdes N, MacFarlane L, Soosairajah J, Bavendiek U, Ahrens I, Ernst S, Bassler N, Missiou A, Patko Z, Aikawa M, Schonbeck U, Bode C, Libby P and Peter K: CD40 ligand mediates inflammation independently of CD40 by interaction with Mac-1. Circulation 100: 1571-1580, 1999. 